\title{
Using LTE in Unlicensed Bands: Potential Benefits and Co-existence Issues
}

\author{
Cristina Cano ${ }^{1}$, David López-Pérez ${ }^{2}$, Holger Claussen ${ }^{2}$, Douglas J. Leith ${ }^{1}$ \\ ${ }^{1}$ Trinity College Dublin, Ireland, ${ }^{2}$ Bell Labs, Alcatel-Lucent, Ireland.
}

\begin{abstract}
Using LTE in unlicensed bands will allow operators to access additional spectrum to meet the increasing demand for mobile services. In this article, we provide an overview of the different approaches currently being considered for LTE operation in unlicensed bands and their interaction with WiFi networks. In summary, LTE-Unlicensed (LTE-U) with carrier sense adaptive transmission is likely to be available in the short term, but cannot be used in all regions due to regulatory restrictions. License Assisted Access (LAA) is intended for use more widely, and so will include listen-before-talk and other features required to conform with, for example, European and Japanese regulations. However, this will require changes to the LTE standards, and so LAA is likely to take longer to deploy. In addition to describing the tradeoffs between these approaches, we also discuss the issue of fair co-existence with existing unlicensed band users, especially WiFi devices.
\end{abstract}

\section{INTRODUCTION}

The use of unlicensed spectrum by mobile network operators, particularly in the $5 \mathrm{GHz}$ band, has recently been attracting considerable attention, and vendors and operators are already actively studying its viability for long term evolution (LTE)/4G cellular networks. The use of the unlicensed spectrum for cellular operation represents a significant change in cellular network deployment and management, and there are, at this stage, still many open questions in terms of both business case and technology as a whole.

Two main approaches to unlicensed LTE are currently being investigated, referred to as LTE-Unlicensed (LTE-U) [1] and Licensed Assisted Access (LAA) [2]. Both augment an existing LTE licensed band interface with unlicensed band transmissions, but LTE-U is a simplified scheme that targets early deployments. LTE-U aims to operate in accordance with the existing Rel. 10/11/12 LTE PHY/MAC standards, and thus does not use listen-before-talk (LBT). However, the absence of LBT restricts its use to regions, such as the US, where this is not required by unlicensed band regulations. LAA is intended for use more widely, and so will include LBT and other features (e.g. minimum bandwidth occupancy, transmit power spectral density) required to conform with, for instance, European and Japanese regulations. Although still under discussion, it seems likely that both of these unlicensed LTE approaches will be used only for transmissions by the base station (downlink transmissions) in first releases, and that most control signalling will be sent via the licensed interface. Extension of LAA to standalone operation (including uplink

CC and DL supported by SFI grants 11/PI/1177 and 13/RC/2077. transmissions), without pairing to a licensed band, is also under consideration.

LTE-U and LAA are particularly attractive to operators that do not own WiFi infrastructure, and are likely to be deployed in very targeted areas using small cells. Initially, it will be an indoor solution in large enterprises and crowded commercial areas like shopping malls, sports arenas, convention centres, where these operators urgently need more capacity. Once the technology matures, it is likely to expand to outdoor hotspots.

A major aspect of ongoing discussions is the requirement to provide fair co-existence with other technologies working in the unlicensed spectrum. Given that current technologies in unlicensed bands, such as $\mathrm{WiFi}$, rely on contention-based access, there is a concern that starvation and other forms of unfairness may occur when they co-exist with a schedule-based technology such as LTE. Currently, two main approaches are under consideration for allowing co-existence when WiFi and LTE nodes share the same channel (see [3], [4]): Carrier Sense Adaptive Transmission (CSAT), which is compatible with existing LTE equipment, and so is suitable for use with LTE-U; and Listen Before Talk (LBT), which requires modification of the current LTE standard, and so is more suitable for use with LAA. In this paper, we describe and evaluate both LTE-U/CSAT and LAA/LBT.

\section{LTE SCHEDULING}

We begin by giving an overview of the LTE fundamentals that impact its deployment in the unlicensed band as well as co-existence with WiFi and with other LTE operators.

\section{A. LTE Framing}

In an LTE wireless cell, time is partitioned into slots of $10 \mathrm{~ms}$ duration, referred to as frames, see Figure 1(a). Each frame is, in turn, subdivided into 10 subframes of $1 \mathrm{~ms}$ duration. Each subframe consists of a set of time-frequency resources, and all LTE transmissions within a cell, both by the basestation and by user equipments (UEs), are assigned to these resources by the LTE basestation scheduler. Scheduling is therefore carried out in a centralised manner within the cell, and all UEs within it must be tightly synchronised in both time and frequency. To assist with managing interference, neighbouring cells and their UEs are also required to maintain tight time synchronisation (typically $\pm 1 \mu \mathrm{s}$ ) so that, for example, a subframe left empty by one cell can be safely re-used by another without interfering with adjacent subframes [5]. 


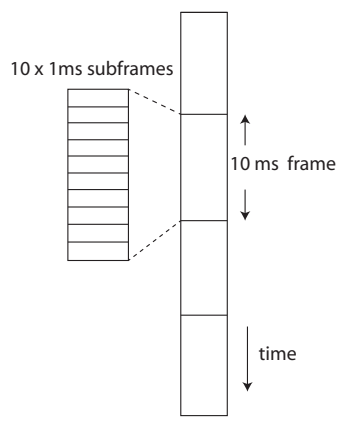

(a) Frames and subframes

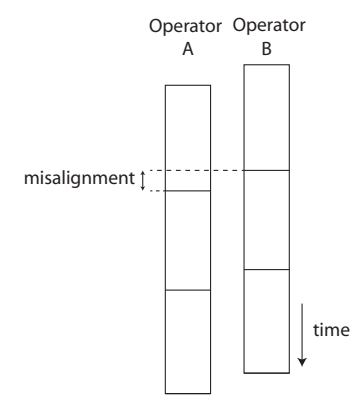

(b) Frame misalignment

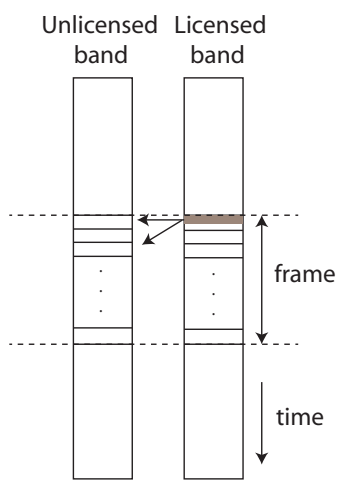

(c) Carrier aggregation

Fig. 1: Illustrating use of frames and subframes for scheduling and carrier aggregation in LTE.

Since LTE was originally designed for operation in licensed bands, the implicit assumption is that the scheduler at the basestation can allocate time-frequency resources without other restrictions. Further, since in the licensed setting, different operators use different spectrum bands, there is no requirement for their frame boundaries to be aligned across networks. That is, transmissions by two different network operators may be misaligned, as illustrated in Figure 1(b).

\section{B. Carrier Aggregation - use of unlicensed spectrum in LTE}

A basestation is not confined to scheduling time-frequency resources within a single spectrum band but rather can simultaneously schedule resources in multiple bands which may be disjoint by means of carrier aggregation [5]. In particular, this can be used to opportunistically augment licensed band transmissions with bandwidth from unlicensed spectrum in the $5 \mathrm{GHz}$ band (and, in due course, also from other unlicensed bands). Using the unlicensed bands for carrier aggregation requires that the licensed interface is always available and active in the basestation.

Such carrier aggregation is illustrated in Figure 1(c). Using cross-scheduling, the control plane information specifying the assignment of transmissions to time-frequency resources and the choice of modulation and coding scheme in each subframe can be transmitted in the licensed band, while the user plane information can be simultaneously transmitted in the unlicensed band, e.g., the shaded area in Figure 1(c) indicates the control plane information in the licensed band and the arrows indicate a scheduling grant in the unlicensed band. Although the control plane information could also be transmitted in the unlicensed band, the great advantage of using the licensed band for transmitting this information is that it avoids the potential corruption by interference on the unlicensed band, e.g., by WiFi transmissions. However, relying on the licensed band for transmission of control plane information requires two active receiving chains at the UE side and the alignment between the frames in the licensed and unlicensed bands, as shown in Figure 1(c).

As already noted, initial deployments of unlicensed LTE are likely to focus on carrier aggregation in the downlink. This implies that ACKs and other transmissions from UEs (uplink transmissions) are also sent using the licensed band.

\section{WIFI SCHEDULING}

WiFi takes a decentralised approach to scheduling transmissions, unlike the centralised approach used by LTE. When a WiFi device wants to make a transmission it senses the radio channel and performs a clear channel assessment (CCA) check. If no transmissions are detected for a period of time (referred to as DIFS) the transmission proceeds. Otherwise the WiFi device draws a number, uniformly at random between 0 and 16 (or between 0 and 32 for $802.11 \mathrm{~b} / \mathrm{g}$ ), and starts to count down while pausing the countdown during periods when the channel is detected to be busy. When the counter reaches zero, a transmission is made. If another device also transmits at the same time then a collision occurs. When a transmission fails (which is detected by the absence of an ACK from the receiver [6]), a new random number is drawn and the process repeats. Usually the interval from which the random number is drawn is doubled on each collision, i.e., increasing as 16, 32, 64 etc. This random access process is referred to as CSMA/CA.

Comparing with LTE, observe that WiFi transmissions are not confined to periodic frame times, and so will generally not be synchronised with LTE transmissions. Further, WiFi defers transmissions when it detects the channel to be busy. It is these two features which make it necessary to take extra measures to ensure that LTE co-exists reasonably fairly ${ }^{1}$ with WiFi when using unlicensed bands. In particular, their use of channel sensing means that WiFi devices might ${ }^{2}$ not start a transmission while LTE is transmitting, and unless LTE leaves idle periods where WiFi devices can access the channel, then WiFi devices may be starved of access. Conversely, since WiFi transmissions occur at random times, these transmissions may overlap with LTE frame boundaries and cause significant interference or cause LTE to refrain from transmission. We discuss LTE/WiFi co-existence in more detail in Section V.

\footnotetext{
${ }^{1}$ It is still unclear which notion of fairness is appropriate in this context. Discussions in the 3GPP are focused on LTE not impacting WiFi performance more than another WiFi network. However, other notions of fairness, such as equal LTE/WiFi throughput or proportional fairness, may well also be of interest.

${ }^{2}$ This depends on the details of the channel sensing used by WiFi and of the signal transmitted by the LTE basestation (especially the received signal strength at WiFi devices), and so it is by no means certain that WiFi devices will always defer to LTE transmissions.
} 


\section{LTE AND WIFI COMPARISON}

One basic question is what advantages and disadvantages unlicensed LTE offers over WiFi. The jury is still out on this, but one important advantage that does seem clear for operators is that network management can be unified by the use of LTE in both licensed and unlicensed bands. For LTE operators without an existing WiFi network, there is also the advantage of access to additional spectrum and bandwidth.

A key issue when discussing these advantages and disadvantages is the throughput and delay performance of LTE and WiFi. With the introduction of new WiFi standards such as IEEE 802.11ac, it is currently difficult to compare these and more work on this is urgently needed. The difficulty is in part because the impact of the requirement for LTE to co-exist with WiFi on performance is not yet clear, but also because the complexity/flexibility of both LTE and WiFi can make simplistic comparisons misleading.

For instance, consider throughput performance. LTE and WiFi use essentially the same physical layer technology, namely OFDM, QAM modulation and MIMO. They do differ in the transmit power and the channel bandwidth, as well as the available choices of modulation and error-correction coding (in 802.11 the minimum rate modulation and coding scheme is BPSK $1 / 2$ while for LTE it is QPSK 78/1024, which affect the coverage range), and the retransmission mechanism on packet loss (WiFi uses ARQ whereas LTE uses hybrid ARQ). Importantly, however, the scheduling, MIMO operation and link adaptation as well as MAC overheads of LTE and WiFi also differ.

In terms of scheduling, LTE uses centralised scheduling whereas WiFi uses random access. Although both approaches can achieve the same throughput capacity as shown in [7], [8], the particular random access approach used by 802.11 results in persistent collisions when more than one device is transmitting. As the number of active devices increases the number of collisions tends to increase, and so network throughput falls. However, in LTE the network throughput also tends to fall as the number of UEs increases since the control plane overhead associated with scheduling transmissions increases. This is, with higher number of UEs, the number of Downlink Control Information (DCI) messages increases, thus, increasing the Control Format Indicator (CFI) value, see for example [9], [10]. This might be mitigated by appropriate scheduling, but such scheduler details are vendor specific.

Nevertheless, and with these caveats in mind, it is possible to quantify the maximum downlink throughput achievable by both technologies in the $5 \mathrm{GHz}$ unlicensed band. We consider the same bandwidth, MIMO configuration and modulation for a channel with high SNR (so that the highest allowed coding rate can be used) and ignoring co-existence requirements (e.g., requirements for LTE channel idle time). We focus on the downlink as LTE unlicensed transmissions are currently confined to this, with uplink transmissions (including ACKs) sent via the licensed band.

As an illustration, consider 64-QAM, a $20 \mathrm{MHz}$ channel and $4 \times 4$ MIMO. For WiFi, the 5/6 coding rate is used and the other MAC parameters are detailed in Table I. For LTE, the coding
TABLE I: IEEE 802.11ac MAC parameters [6]

\begin{tabular}{|c|c|}
\hline Slot Duration $(\sigma)$ & $9 \mu \mathrm{s}$ \\
\hline DIFS & $34 \mu \mathrm{s}$ \\
\hline SIFS & $16 \mu \mathrm{s}$ \\
\hline PLCP Preamble+Headers Duration $\left(T_{\mathrm{plcp}}\right)$ & $40 \mu \mathrm{s}$ \\
\hline PLCP Service Field $\left(L_{\mathrm{s}}\right)$ & 16 bits \\
\hline MPDU Delimiter Field $\left(L_{\mathrm{del}}\right)$ & 32 bits \\
\hline MAC Header $\left(L_{\mathrm{mac}-\mathrm{h}}\right)$ & 288 bits \\
\hline Tail Bits $\left(L_{\mathrm{t}}\right)$ & 6 bits \\
\hline ACK Length $\left(L_{\mathrm{ack}}\right)$ & 256 bits \\
\hline Payload $(D)$ & 768000 bits $(64 \times 1500 \mathrm{~B})$ \\
\hline
\end{tabular}

TABLE II: Comparison of the peak throughput of LTE and WiFi (5GHz band, $20 \mathrm{MHz}$ channel, 64-QAM, 5/6 (IEEE 802.11ac) and 948/1024 (LTE) coding rate, 4x4 MIMO).

\begin{tabular}{|c|c|}
\hline IEEE 802.11ac & $236.9 \mathrm{Mbps}$ \\
\hline \hline LTE $($ CFI $=0)$ & $373.3 \mathrm{Mbps}$ \\
\hline LTE $($ CFI $=1)$ & $300.8 \mathrm{Mbps}$ \\
\hline LTE $($ CFI $=2)$ & $283.2 \mathrm{Mbps}$ \\
\hline LTE $($ CFI $=3)$ & $256.6 \mathrm{Mbps}$ \\
\hline
\end{tabular}

rate of 948/1024 is used and a range of values for the minimum CFI are considered. The goal is to assess whether for the same conditions the use of LTE provides substantially higher throughput performance. However, note that WiFi currently allows for a larger number of aggregated channel widths which provides a higher peak throughput performance.

The peak throughput is shown in Table II for both LTE and WiFi. Observe that LTE offers a $27 \%$ increase in throughput compared to WiFi IEEE 802.11ac when the CFI equals 1, falling to $7 \%$ when the CFI equals 3 . When the licensed band is used for the LTE downlink control plane, then the associated CFI overhead in the unlicensed band falls to zero, and the LTE unlicensed band throughput rises correspondingly to $373.3 \mathrm{Mbps}$ which is $57.6 \%$ higher than WiFi. Once again it should be borne in mind that even this idealised comparison is still not really "fair" since it neglects the LTE control plane overheads in the licensed band. However, the results obtained do not seem to provide substantially higher throughput so as to justify the use of unlicensed LTE compared to WiFi. Nevertheless, as we stated before, the unification of network management can instead be a strong motivating factor for unlicensed LTE roll-out.

\section{LTE/WIFI AND LTE/LTE CO-EXISTENCE}

An important constraint on LTE unlicensed band operation is the requirement for efficient co-existence with other unlicensed band users, in particular with WiFi users and different LTE operators. Co-existence of multiple LTE operators within the same unlicensed band is a major concern, although one that has received only limited attention to date. Instead, the main focus until now has been on LTE/WiFi co-existence, due to the large volume of already deployed WiFi nodes ${ }^{3}$.

In this section, a survey of current LTE/WiFi co-existence mechanisms is presented.

\footnotetext{
${ }^{3}$ In the US a number of WiFi operators have already expressed their concerns and approached regulatory government bodies indicating that LTE-U and LAA operations may have a detrimental impact on existing and future use of unlicensed or shared spectrum.
} 


\section{A. Channel selection}

Perhaps the simplest approach to co-existence is to ensure that WiFi and LTE devices use different channels that do not interfere with one another. As shown, for example, in [11], efficient selection of non-interfering channels is feasible, and can be realised using decentralised algorithms that do not require explicit communication among nodes. The $5 \mathrm{GHz}$ band has a relatively large number of non-overlapping $20 \mathrm{MHz}$ channels, which simplifies such channel selection. However, in certain locations such as the US, with four $20 \mathrm{MHz}$ channels in the U-NII-1 band and five $20 \mathrm{MHz}$ channels in the U-NII-3 band, it may be challenging to find clear channels.

\section{B. Power control}

Adjustment of the transmission power of the cell might also be used to assist co-existence by reducing interference. However, simply reducing transmit power adversely affects cell coverage and data rate, and so more sophisticated schemes, which adapt power per carrier and sub-carrier (each $20 \mathrm{MHz}$ channel is divided into a number of narrow-band sub-carriers) to achieve a targeted coverage/performance are also of interest.

\section{Discontinuous transmission}

In the event that channel selection and power control is not sufficient to avoid interference, LTE can use discontinuous transmission. That is, rather than using every available timefrequency resource to make a transmission, some slots are left blank for the sake of interference coordination. While in principle each time-frequency resource might be considered independently, in practice attention has mainly focused on leaving subframes or entire frames blank.

Recall that one basic difference between the two technologies, LTE and WiFi, is that LTE unlicensed band transmissions must be aligned with fixed frame/subframe boundaries, see Figure 1(c), whereas WiFi transmissions are not subject to this constraint. Another is that WiFi defers transmissions when it detects the channel to be busy. With these in mind, the main approaches currently under consideration for ensuring coexistence when LTE and WiFi nodes share the same channel are discussed in the following.

1) LTE-U/Carrier Sense Adaptive Transmission (LTEU/CSAT): One approach is LTE-U/CSAT [3], [4], which is mainly targeted at early deployments and for the US market where LBT is not required. In this approach, an LTE basestation schedules transmissions periodically, leaving idle times between transmissions to allow WiFi devices to transmit. For example, the basestation may transmit on every other frame boundary so that it transmits one $10 \mathrm{~ms}$ frame and then leaves the channel idle during the next $10 \mathrm{~ms}$ frame, yielding a $50 \%$ on-off duty cycle, see Figure 2 . In order to enhance performance, subframe granularity is desired.

To implement LTE-U/CSAT, the existing almost blank subframes (ABS) framework of LTE was initially considered in [12]. However, since synchronisation signals and control information are still present in ABS, there may still be an impact on WiFi transmissions and carrier sensing. Instead, the MAC channel element activation and deactivation feature of carrier

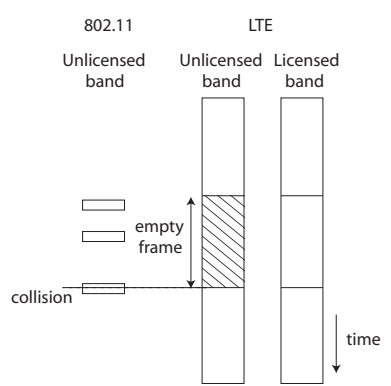

(a) LTE-U/CSAT

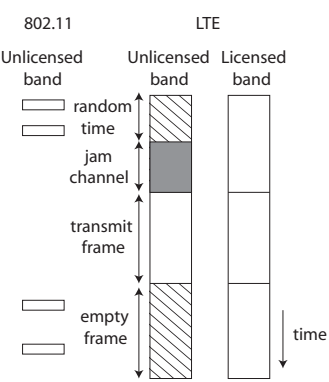

(b) LAA/LBE
Fig. 2: Illustrating main LTE co-existence approaches.

aggregation has been adopted by the LTE-U Forum [13], which is compatible with Rel. 10/11/12 LTE PHY/MAC standards. In this way, the LTE basestation can activate and deactivate the unlicensed carrier in the UE. The LTE basestation may sense the channel during the idle times in order to adapt the on-off duty-cycle so as to leave more or less time for $\mathrm{WiFi}$ transmissions.

Note that a WiFi transmission may start towards the end of a LTE-U/CSAT idle period, and so overlap with the start of an LTE transmission due to the absence of channel sensing, as illustrated in Figure 2(a) (where it is marked "collision"). Such collisions at the start of an LTE transmission essentially reduce both LTE and WiFi throughput (even if colliding transmissions can be decoded, which may be far from straightforward, the information rate is necessarily reduced). For a given on-off duty cycle, this reduction in throughput can be minimised by making the LTE transmission as long as possible, thus reducing the collision probability, but of course this comes at the cost of increased delay for all devices. A throughtputdelay trade-off therefore exists.

To illustrate these effects, we show in Figure 3 the WiFi and LTE throughput obtained from simulations when we vary the number of subframes transmitted at each LTE busy period. We have implemented the specific modules for LTE and WiFi channel access in an event-based simulator framework that provides a time reference, modules and interconnection capabilities. The same parameters shown in Table I are used for the WiFi network, and the physical parameters used for the throughput comparison in Table II (with $\mathrm{CFI}=0$ ) are used for the LTE network. We provide simulation results for different scenarios where $n$ denotes the number of WiFi users. In more detail, we consider two scenarios with a WiFi access point providing service to a single WiFi user $(n=1)$ or five WiFi users $(n=5)$, while sharing the channel with an LTE basestation providing service to one UE. As a baseline, we also show the throughput obtained when the LTE basestation is replaced by a WiFi access point (labelled as WiFi only $n=2$ and $n=6$, respectively - the LTE UE becomes a WiFi user $)^{4}$. The on-off duty cycle of the LTE basestation is configured at $50 \%$, we consider saturated conditions and ideal WiFi carrier sensing (WiFi stations always defer upon

\footnotetext{
${ }^{4}$ Note that in the WiFi only case, performance is no longer dependent on the number of LTE subframes per channel access since there is no LTE transmission.
} 
detection of LTE transmissions). The LTE basestation, WiFi access point and users are all within coverage range, thus, no hidden terminal problems arise. Collisions, both between WiFi stations and among $\mathrm{WiFi}$ and LTE, are considered. We also assume that only LTE subframes which do not overlap with WiFi transmissions contribute to the LTE throughput, which represents a lower bound. The main goal of the simulation setup is to provide a controlled scenario that allows insight into the effect of the LTE transmission duration.

Figure 3 shows that when the LTE idle period duration is smaller than a WiFi transmission, which lasts approximately $3 \mathrm{~ms}$, WiFi does not have any opportunity to access the channel and thus the resulting WiFi throughput is zero. When the LTE idle period duration increases, it can be seen that the throughput for both technologies tends to increase as $\mathrm{WiFi}$ has room to access the channel and the collision probability becomes smaller. Note also the zig-zag effect that depends on the value of the number of subframes transmitted per LTE channel access. This is a quantisation effect associated with the number of WiFi transmissions that can completely fit within an LTE idle period. Importantly, Figure 3 shows that to optimise throughput the duration of this idle period should be carefully selected, and we should consider that longer on and off period durations tend to reduce collision probability between both technologies. However, a long on and off period duration, such as $20 \mathrm{~ms}$, significantly increases the delay variability for both WiFi and LTE, and it is not yet clear how sporadic long delays may affect TCP and higher layer dynamics, or whether issues may arise such as causing a WiFi station to de-associate from its access point.

It is also important to note in Figure 3 that when the LTE basestation and WiFi access point are serving the same number of users and traffic, (this is the case for $n=1$ ) and the LTE busy period duration is similar to the WiFi transmission duration (immediately longer than 3 subframes per channel access), the throughput obtained per user, both for LTE and $\mathrm{WiFi}$, is comparable. However, when $n=5$, we can observe that no configuration provides similar user throughput for LTE and WiFi since there are 5 times less LTE users than WiFi ones. Therefore, a reduced duty cycle with a larger LTE idle period duration needs to be used to leave more transmission opportunities to $\mathrm{WiFi}$, if the goal is to achieve equal throughput between $\mathrm{WiFi} / \mathrm{LTE}$ users.

With regard to co-existence as defined in [2], we can also derive from Figure 3 the value of the on and off period durations that should be used for LTE to impact WiFi throughput in a similar manner as another WiFi. For $n=1$, where the LTE basestation and WiFi access point are serving the same number of users and traffic and the same channel time is given to both technologies (the on-off duty cycle of the LTE basestation is configured at $50 \%$ ), this co-existence is achieved at different configurations, the one with shorter LTE on and off durations being 7 LTE subframes. However, in order to further improve throughput, we can achieve this co-existence metric at longer LTE on and off durations, at the expense of WiFi delay increased variability. For durations equal or larger than 20 subframes, we can say that LTE-U/CSAT always achieves this co-existence target and the throughput of both technologies is

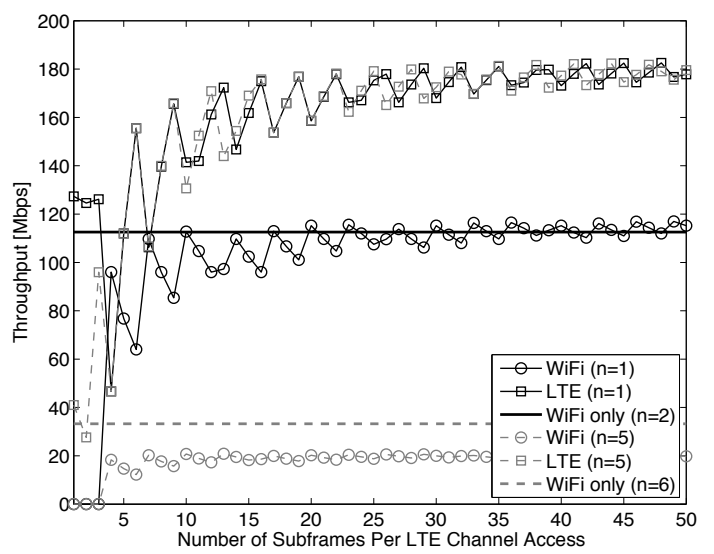

Fig. 3: Per-station/UE throughput for WiFi and LTE using LTE-U/CSAT with $n=1$ and $n=5 \mathrm{WiFi}$ users. LTE throughput is the aggregate over all UEs.

maximised since the collision probability is reduced. However, as for the equal throughput case, for $n=5$, we observe that a larger idle period duration is needed to achieve this coexistence target since there are 5 times less LTE users than WiFi ones. Thus, both the duty cycle and the duration of the LTE on and off period durations are key parameters for providing a certain metric of fairness.

2) LAA/Listen Before Talk (LAA/LBT): An alternative to LTE-U/CSAT is LAA/LBT, in which the LTE basestation senses the channel using energy detection within a designated time before starting transmissions in the unlicensed band. Such sensing is mandatory in regions such as Europe and Japan.

LAA/LBT approaches can be classified according to various categories, but the most relevant is LBT-Load Based Equipment (LBE) Category 4: LBT with random back-off and variable size of contention window (see Section 8.2 of [2]). This is similar to the random access procedure used by WiFi devices and is recommended by the 3GPP as the baseline approach for LAA downlink transmissions.

A significant advantage of using a similar random access procedure to 802.11 devices to win transmission opportunities is that fair co-existence with 802.11 devices can be more easily guaranteed. Importantly, co-existence of multiple LTE networks within the unlicensed band can also be ensured in a more straightforward manner.

When using LAA/LBT-LBE, data transmission can start right after the carrier sensing procedure. However, when a transmission opportunity is obtained, it will, of course, not usually be aligned with an LTE frame boundary, and LTE devices cannot start transmissions until the next frame boundary is reached. To hold onto the channel and prevent WiFi devices from starting transmissions, the LTE basestation may transmit a reservation signal causing $\mathrm{WiFi}$ devices to detect the channel as being busy and so defer their transmissions. This is illustrated in Figure 2(b). A disadvantage is that the use of this reservation signal carries an overhead since no 


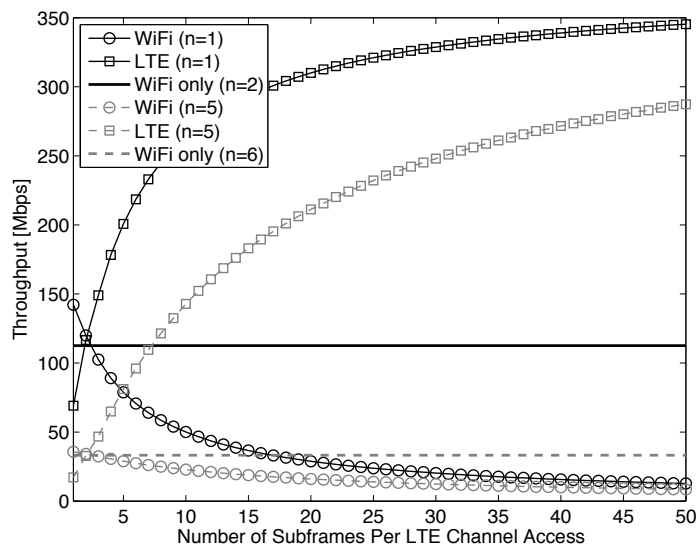

(a) Per-station/UE throughput

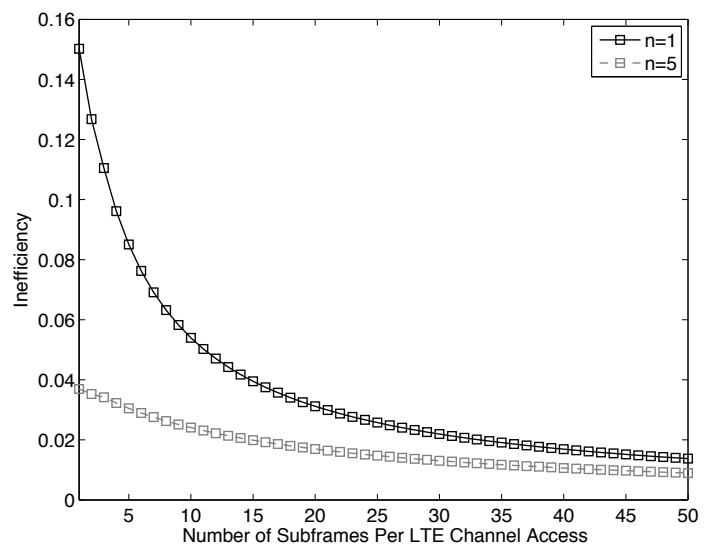

(b) Inefficency

Fig. 4: Performance for WiFi and LTE using LAA/LBT-LBE with $n=1$ and $n=5 \mathrm{WiFi}$ users. LTE throughput is the aggregate over all UEs.

device can transmit data during this period ${ }^{5}$, and this overhead can significantly impact network throughput. This signal, however, may carry control information to assist automatic gain control/synchronisation, broadcast control information or enhanced PDCCH, thus minimising the waste of resources. Note that transmitting no reservation signal may result in a large number of unsuccessful transmission attempts, since a WiFi node may start transmission before the subframe boundary is reached.

Figure 4(a) shows the throughput for LTE and WiFi using LAA/LBT-LBE with the same parameters and configurations used in the last subsection. It can be observed here that the number of LTE subframes per channel access that LTE should transmit to provide equal LTE/WiFi throughput as well as to impact WiFi as another WiFi network are similar. Note that, in this case, the value of the LTE active time to achieve both objectives is around $3 \mathrm{~ms}$, which is approximately the duration of a WiFi transmission in the considered scenario. Basically, since LTE is using the same access method, i.e., LAA/LBT

${ }^{5}$ In order to deal with this inefficient use of channel resources, the $3 \mathrm{GPP}$ is looking at the definition of a more flexible Transmission Time Interval (TTI) where transmissions do not need to start at the subframe boundary or to be confined to one subframe. with random back-off and variable size of contention window, and channel access parameters as $\mathrm{WiFi}$, co-existence as defined above is achieved when LTE and WiFi grab the channel for the same amount of time, in this case, $3 \mathrm{~ms}$.

Figure 4(b) also shows the percentage of channel resources used for transmitting the reservation signal. Note the tradeoff between efficiency and the impact on WiFi performance. While increasing the transmission duration of LTE decreases the inefficiency caused by the transmission of the reservation signal, the WiFi throughput degrades considerably since both LTE and WiFi have the same channel access opportunities, but WiFi only accesses the channel for $3 \mathrm{~ms}$ (the transmission duration of $\mathrm{WiFi}$ is upper bounded by the maximum number of packets that can be aggregated in a single transmission and by the maximum allowed duration of a packet/burst transmission [6]).

\section{SCOPE AND Future DiRECTIONS}

In our evaluation we have made a number of considerations. In this section we examine these assumptions in detail and discuss associated future research directions.

(i) Unsaturated stations: In our evaluation, we have assumed that the $\mathrm{WiFi}$ and unlicensed LTE stations are saturated, i.e. there is always a packet buffered for transmission. Note that coexistence between unlicensed LTE and WiFi is more problematic when the networks have to cope with high traffic demands. From the point of view of LTE, this seems a valid assumption, as otherwise the licensed band will suffice. It also seems probable that the activity of the LTE network will result in saturation of the coexisting WiFi stations. However, further work considering a broad range of offered loads and traffic distributions, both for LTE and WiFi, might be in place in order to understand the complex dynamics of these interactions and their impact on coexistence. (ii) Perfect WiFi Carrier Sensing of LTE: We assume in this work that WiFi defers its channel access attempts when the medium is busy due to LTE transmissions. At this point, it is still not clear under which conditions WiFi carrier sense fails to detect LTE transmissions and extensive experimental works on this should be carried out. It is important to point out that mechanisms such as the CTS-to-self [14] can be used in LTE to ensure WiFi reliably detects LTE transmissions. (iii) Hidden Terminals: The basic difficulties here arise from the fact that the effects of hidden terminals are highly dependent on the scenario of evaluation and an extensive evaluation is required in order to obtain meaningful conclusions. It is perhaps also worth noting here that the prevalence of severe hidden terminals in real network deployments presently remains unclear, especially in small cell scenarios. (iv) Multiple LTE networks: We have considered in our evaluation that only one LTE network is active in a given channel. The consideration of multiple LTE networks sharing the unlicensed spectrum has challenges associated related to the misalignment of the subframes boundaries among networks belonging to different operators. Note that given this, results are extremely dependent on the magnitude of that $d e$ synchronisation and that an extensive evaluation should be carried out to shed some light on this. However, although this 
consideration is interesting from a scientific point of view, it is not clear whether in practice multiple LTE networks will be configured to select a channel already in use by another unlicensed LTE operator.

\section{FINAL REMARKS}

The use of unlicensed band spectrum to opportunistically augment licensed band transmissions potentially offers great benefits for cellular operators. In addition to access to additional spectrum and bandwidth, and thus capacity, network management can be unified by use of LTE in both licensed and unlicensed bands, streamlining authentication, handover and resource allocation. However, these benefits for cellular operators should not be realised at the expense of other technologies operating in the unlicensed band. Simulations results presented in this paper shed some light on this, and show that LTE-U/CSAT and LAA/LBT can impact WiFi throughput in a similar manner as another WiFi if they are properly designed and configured. For LTE-U/CSAT, the duty cycle and the duration of the on and off periods are key parameters for achieving co-existence, which should be tuned according to the number of neighbouring WiFi devices to achieve the desired metric of fairness and minimise collisions with ongoing WiFi transmissions. For LAA, the adoption of LAA/LBT with random back-off and variable size of the contention window as well as a maximum channel occupancy time similar to $\mathrm{WiFi}$ is key for easily reaching co-existence between technologies.

First commercial LTE-U small cell products are expected to start becoming available on the market by the second half of 2016, although efforts to enhance the performance of LTEU/CSAT as a co-existence method are also expected to continue. In parallel, the 3GPP TSG-RAN group finalised the first LAA specification in March 2016 as part of LTE Rel. 13, and LAA commercial products are expected to follow in 2017. The standardisation work on LAA continues in LTE Rel. 14, where the major efforts are on enhancements to support aggregation for uplink transmissions. The standardisation teams are also working on co-existence issues. The 3GPP, the IEEE and the Wi-Fi Alliance are working together to finalise the LAA design features and are discussing technical aspects, mostly related to LAA/LBT, which may impact co-existence.

\section{REFERENCES}

[1] Alcatel-Lucent, Ericsson, Qualcomm, Samsung, and Verizon, "LTE-U Technical Report Coexistence Study for LTE-U SDL V1.0," LTE-U Forum, Tech. Rep., Feb. 2015. Accessed on 10/06/2016.

[2] 3GPP TR 36.889, "Feasibility Study on Licensed-Assisted Access to Unlicensed Spectrum,” v.13.0.0, 2015. Accessed on 10/06/2016.

[3] M. I. Rahman, A. Behravan, H. Koorapaty, J. Sachs, and K. Balachandran, "License-exempt LTE systems for secondary spectrum usage scenarios and first assessment," in IEEE Symposium on New Frontiers in Dynamic Spectrum Access Networks (DySPAN), 2011, pp. 349-358.

[4] Qualcomm Technologies, Inc., "LTE in Unlicensed Spectrum: Harmonious Coexistence with Wi-Fi," Whitepaper, June, 2014. Accessed on 10/06/2016.

[5] S. Sesia, I. Toufik, and M. Baker, Eds., The UMTS Long Term Evolution: From Theory to Practice, 2nd ed. Wiley \& Sons, Feb. 2009.

[6] IEEE Std 802.11ac, "Wireless LAN Medium Access Control (MAC) and Physical Layer (PHY) Specifications. Amendement 4: Enhancements for Very High Throughput for Operation in Bands below $6 \mathrm{GHz}$," ANSI/IEEE Std 802.11ac, 2013.
[7] J. Barcelo, B. Bellalta, C. Cano, and M. Oliver, "Learning-BEB: Avoiding Collisions in WLAN," in Eunice Summer School, 2008.

[8] M. Fang, D. Malone, K. Duffy, and D. Leith, "Decentralised Learning MACs for Collision-free Access in WLANs," Wireless Networks, 2012.

[9] D. Lopez-Perez, M. Ding, H. Claussen, and A. H. Jafari, "Towards 1 Gbps/UE in Cellular Systems: Understanding Ultra-Dense Small Cell Deployments," Arxiv preprint arXiv:1503.03912, 2015.

[10] M. Ding, P. Wang, D. Lopez-Perez, G. Mao, and Z. Lin, "Performance Impact of LoS and NLoS Transmissions in Small Cell Networks," Arxiv preprint arXiv:1503.04251, 2015.

[11] D. Leith, P. Clifford, V. Badarla, and D. Malone, "WLAN channel selection without communication," Computer Networks, vol. 56, no. 4, pp. 1424-1441, 2012.

[12] E. Almeida, A. Cavalcante, R. Paiva, F. Chaves, F. Abinader, R. Vieira, S. Choudhury, E. Tuomaala, and K. Doppler, "Enabling LTE/WiFi coexistence by LTE blank subframe allocation," in IEE Conference on Communications (ICC), 2013, pp. 5083-5088.

[13] Alcatel-Lucent, Ericsson, Qualcomm, Samsung, and Verizon, "LTEU CSAT Procedure TS V1.0," LTE-U Forum, Tech. Rep., Oct. 2015. Accessed on 10/06/2016.

[14] A. K. Sadek, T. Kadous, K. Tang, H. Lee, and M. Fan, "Extending LTE to unlicensed band-Merit and coexistence," in Communication Workshop (ICCW), 2015 IEEE International Conference on, 2015. 